\title{
Elaboración de un platillo aplicando la técnica de esferificación
}

\section{Preparation of a dish using the spherification technique}

\author{
Tania Hernández Sánchez ${ }^{a}$, Alexia Vázquez Hernández, ${ }^{b}$ \\ Juan Francisco Gutiérrez Rodríguez ${ }^{c}$
}

\begin{abstract}
:
Currently one of the culinary innovations is to use unconventional ingredients in dishes to recreate emblematic dishes of the kitchens, this is how hot jellies, foams and spherifications cause amazement and surprise in diners. These techniques are one more tool that gourmets integrate into their culinary work to communicate while exalting specific properties in the ingredients to enrich their food.
\end{abstract}

Among one of the most used are spherifications, a technique that allows the controlled gelation of a liquid that is mixed with sodium alginate to transform it into spheres, gelled inside.

Keywords:

Innovation, spherification, techniques, food, molecular

\section{Resumen:}

Actualmente una de las innovaciones culinarias es el utilizar en platillos ingredientes no convencionales para recrear platillos emblemáticos de las cocinas, es así como las gelatinas calientes, las espumas y esferificaciones causan asombro y sorpresa en los comensales. Estas técnicas son una herramienta más que los gastrónomos integran en su quehacer culinario para comunicar al mismo tiempo que exaltan propiedades especificas en los ingredientes para enriquecer sus alimentos.

Entre una de las más utilizadas destacan las esferificaciones, técnica que permite la gelificación controlada de un líquido que es mezclado con alginato de sodio para transformarlo en esferas, gelificadas en su interior.

Palabras Clave:

Innovación, esferificación, técnicas, alimentos, molecular

\section{Introducción}

La esferificación es una técnica culinaria que permite la gelificación controlada de un líquido que es mezclado con alginato de sodio para transformarlo en esferas, que, gelificadas por dentro, comúnmente para lograr este efecto se debe verter la mezcla en un baño con cloruro cálcico. 1

Con la esferificación desea imitarse una forma, y textura, muy similar a las huevas de pescado, para que, con el encapsulamiento los sabores aparezcan repentinamente

a Autor de Correspondencia, Profesor de la Licenciatura de Gastronomía, Universidad Autónoma del Estado de Hidalgo, https://orcid.org/0000-0002-2119-8381, Email: tania.hernandez@uaeh.edu.mx

b Autor de Correspondencia, Profesor de la Licenciatura de Gastronomía, Universidad Autónoma del Estado de Hidalgo, https://orcid.org/0000-0001-6973-7009, Email: alexia_vazquez@uaeh.edu.mx

c Profesor de la Licenciatura de Gastronomía, Universidad Autónoma del Estado de Hidalgo, https://orcid.org/0000-0002-4225-563X , Email: juanfg@uaeh.edu.mx 
en la boca, como pueden ser vinos, zumos de frutas o verduras. El propósito de la esferificación es modificar las propiedades texturales de los alimentos para así desarrollar nuevas sensaciones en el comensal. 2

Los alimentos que no se recomiendan para este procedimiento son líquidos-lácteos, líquidos-grasos y líquidos con alto porcentaje de alcohol.1

Se diferencian dos procesos de esferificación:

- Esferificación directa: La esferificación se lleva a cabo mezclando el líquido alimenticio con alginato de sodio y dejándolo gotear sobre una disolución fría de cloruro de calcio o carbonato de calcio

- Esferificación inversa: Sí el líquido que se desea esterificar ya contiene calcio, se sumerge en una disolución de alginato para producir las esferas. Pero si el líquido no posee calcio se le añade gluconolactato de calcio y se procede de igual forma.

En ambos casos lo que se obtiene es una gota de líquido contenida dentro de una delgada membrana esférica. 3,4,5

Por lo cual el objetivo de la práctica es realizar un platillo utilizando la técnica de esferificación para mejorar la experiencia sensorial del comensal.

\section{Desarrollo}

a) Nombre de la práctica: Gnocchi de papa

b) Insumos requeridos para su realización

\begin{tabular}{|c|c|c|}
\hline INGREDIENTES & CANTIDAD & UNIDAD \\
\hline CEBOLLA & 0.250 & pieza \\
\hline $\begin{array}{l}\text { ACEITE } \quad \text { DE } \\
\text { OLIVA }\end{array}$ & $\mathrm{c} / \mathrm{s}$ & \\
\hline $\begin{array}{l}\text { DIENTES DE } \\
\text { AJO }\end{array}$ & 2 & piezas \\
\hline $\begin{array}{l}\text { PAPA } \quad \text { EN } \\
\text { CUBOS }\end{array}$ & 1 & pieza \\
\hline FONDO DE AVE & 0.500 & $\mathrm{Lt}$ \\
\hline $\begin{array}{l}\text { CONDIMENTO } \\
\text { PARA PAELLA }\end{array}$ & 1 & sobre \\
\hline SAL & $\mathrm{c} / \mathrm{s}$ & \\
\hline ALGIN & 8 & gr por Lt \\
\hline CALCIC & 6 & gr por Lt \\
\hline
\end{tabular}

\section{c) Procedimiento}

Saltear la cebolla y el ajo finamente picados, agregar el fondo y las papas en cubos., condimento. Cocer todo tapado hasta que las papas se hayan cocinado, licuar y colar. Sazonar, enfriar antes de agregar el químico, incorporar el alginato y perfumar con el aceite y colar en manta de cielo. Con la ayuda de una cucharita realizar los gnocchis sobre el agua que tendrá el calcic dejar que el químico haga su función. Sacar con cuidado y con la ayuda de una cuchara perforada, enjuagar y reservar para el momento de servir pasar por agua caliente.

\section{Conclusión}

La transformación de la comida en presentaciones convencionales permite presentar platos con toques sorprendentes y lúdicos. Las esferificaciones son técnicas culinarias que permiten ser utilizadas para la elaboración de platillos innovadores de tipo entradas, fondos y bebidas.

\section{Referencias}

[1] Morales, A. (2017). Caracterización de la esterificación funcional de alginato, goma xantana y pulpa de guanábana. Agosto, 2021, de Universidad Autónoma Agraria Antonio Narro Sitio web: http://repositorio.uaaan.mx:8080/xmlui/bitstream/handle/123456789/4 2109/K\%2064607\%20ISAMAR\%20ALEJANDRA\%20MORALES\% 20IBARRA.pdf?sequence $=1$ \&isAllowed $=y$

[2] Corell, P., García, P., Sapiña, F., Farré, I. Castells, P., Martínez, J.(2007). Efecto del ión calcio sobre las propiedades texturales de esferas de alginato aplicadas a cocina. Alimentaria: Revista de tecnología e higiene de los alimentos, 388, 79-83.

[3] Mans, C. (2014). Sferificaciones y macarrones. La ciencia en la cocina tradicional y moderna. Barcelona: Ariel.

[4] Lupo Pasin, B. (2014). "Estudio de la gelificación de alginatos para encapsulación: caracterización, preparación y aplicaciones en alimentos funcionales". Barcelona, España: Universitat de Barcelona.

[5] Alva, C. R. (2019). Esferificación a la inversa para incrementar la diversidad gastronómica, Chachapoyas, 2018. Revista de Investigación Científica UNTRM: Ciencias Sociales y Humanidades, 2(1), 49-54. 